\title{
Minority charge carrier lifetime mapping of crystalline silicon wafers by time-resolved photoluminescence imaging
}

\author{
David Kiliani, ${ }^{\text {a) }}$ Gabriel Micard, Benjamin Steuer, Bernd Raabe, Axel Herguth, \\ and Giso Hahn \\ University of Konstanz, Department of Physics, Konstanz 78457, Germany
}

(Received 11 May 2011; accepted 21 July 2011; published online 8 September 2011)

\begin{abstract}
A camera-based method to record spatially and time-resolved photoluminescence images of crystalline silicon wafers was developed. The camera signal is modulated by a rotating shutter wheel, allowing for a wide range of camera types to be used for the measurement and easy integration into existing photoluminescence setups. The temporal resolution is sufficient to record the decay curve of photoexcited charge carriers in surface-passivated silicon wafers. A transient measurement of minority carrier lifetimes down to less than $10 \mu$ s can be obtained for each pixel individually, without the need for any external calibration.

[doi:10.1063/1.3630031]
\end{abstract}

\section{INTRODUCTION}

In the last years, camera-based photoluminescence imaging (PLI) has become a widely used characterization method for crystalline silicon wafers and solar cells. ${ }^{1-5}$ It provides high spatial resolution with short measurement time and does not require any contacting of the sample. Due to the correlation between photoluminescence (PL) signal and excess carrier density $\Delta n,{ }^{6}$ the PLI method can be used for a spatially resolved measurement of the effective excess minority charge carrier lifetime $\tau_{\text {eff }}$ using the steady-state condition $\Delta n=G \tau_{\text {eff. }}{ }^{7}$ However, this requires a calibration of the setup to determine the generation rate $G$ and the exact correlation between PL signal and $\Delta n$. An integral method can be used for calibration, ${ }^{2,3}$ but lateral variations of the calibration factors, e.g., caused by changes in surface texture or grain orientation, can lead to incorrect values of $\tau_{\mathrm{eff}} .^{3}$

Dynamic carrier lifetime measurements do not depend on this absolute intensity calibration. They are usually done either by integral methods, like quasi-steady-state photoconductance $^{8}$ (QSSPC) or photoluminescence, ${ }^{9}$ (QSSPL) or with scanning methods, like microwave-detected photoconductance decay ${ }^{10}$ (MW-PCD), where the measurement is performed sequentially for each point of the sample. Recently, a time-resolved, camera-based lifetime mapping method measuring free carrier absorption (CDI/ILM) has been demonstrated. ${ }^{11}$ The thermo-sensitive camera $(4-5 \mu \mathrm{m})$ used by Ramspeck et al. ${ }^{11,12}$ is capable of very short exposure times without an external shutter. These short exposure times are not possible with high-resolution silicon charge-coupled device (CCD) sensors commonly used for PLI. Indium gallium arsenide (InGaAs) complementary metal-oxide-semiconductor (CMOS) cameras can also be used for PLI. CMOS camera sensors feature a signal amplifier for each pixel, allowing for electronic shuttering, which is fast enough for dynamic lifetime mapping of crystalline silicon wafers. ${ }^{13}$ However, the image quality of silicon CCD cameras is usually much better than the one of InGaAs CMOS cameras, at considerably lower costs.

${ }^{a) E l e c t r o n i c ~ m a i l: ~ d a v i d . k i l i a n i @ u n i-k o n s t a n z . d e . ~}$
The time-resolved photoluminescence imaging (TRPLI) method presented in this work is therefore based on an external rotating shutter wheel. This approach combines the advantages of time-resolved imaging with the higher spatial resolution and lower equipment cost of CCD sensors.

\section{MEASUREMENT SETUP AND PROCEDURE}

The photoluminescence setup is schematically shown in Fig. 1. It consists of the camera with a longpass filter (a), a rotating shutter wheel with slits (c), two objective lenses (b and d) and the sample (f), which is placed on top of a light-emitting diode (LED) panel (g). The LED panel homogeneously illuminates the sample from the backside with an adjustable photon flux $E$ and can be switched on and off by an excitation control box (e). A relatively short LED wavelength of $630 \mathrm{~nm}$ was chosen to minimize the amount of LED light emitted in the PL signal range of $900 \mathrm{~nm}$ to $1100 \mathrm{~nm}$. An additional shortpass filter on top of the LEDs blocks the remaining emission at these wavelengths. A laser illumination system could also be used instead of the LED panel, provided the laser intensity can be modulated fast enough.

The first objective lens (d) creates an intermediate image of the luminescence radiation of the sample. The shutter wheel (c) rotates in the image plane of this intermediate image and periodically interrupts the focused luminescence radiation with a 1:1 mark to space ratio. The shuttered intermediate image is then projected onto the CCD sensor of the camera (a) with a second objective lens (b).

This setup significantly differs from the one used in earlier TR-PLI measurements. ${ }^{14}$ All luminescence light emitted at a point $i$ of the sample is focused to a point $i^{\prime}$ on the intermediate image plane. By placing the shutter wheel at this intermediate image plane, the whole signal from $i$ is blocked instantaneously as soon as the shutter wheel edge passes through $i^{\prime}$. Therefore, the blurring of the shutter wheel transmit function $H(t)$ reported previously ${ }^{14}$ disappears.

An iKon-M DU934 N-BR-DD camera from Andor Technologies was used in this setup, which features a backilluminated 1megapixel silicon CCD chip with high sensitivity 


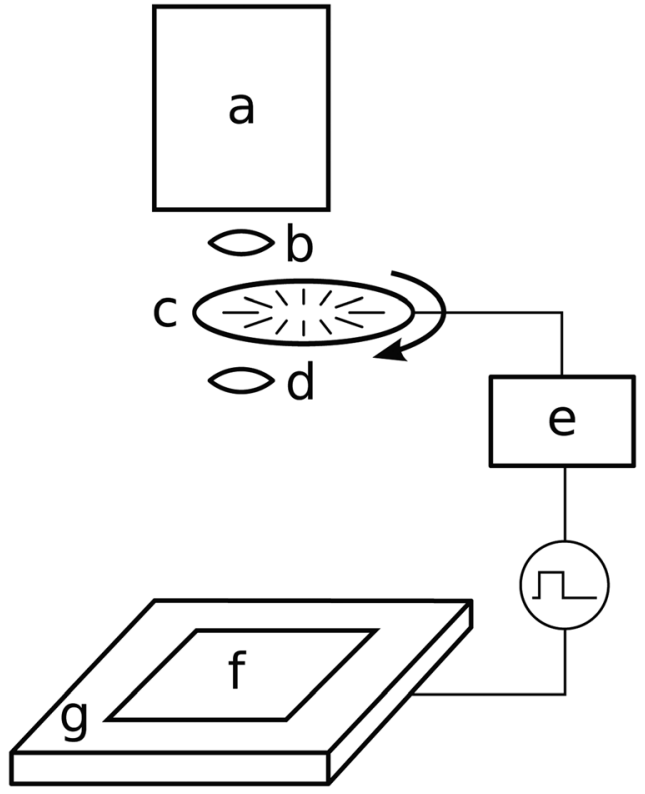

FIG. 1. Schematic view of the measurement setup. The sample (f) is illuminated by a LED panel ( $\mathrm{g}$ ) and emits PL light, which is focused to a shutter wheel (c) by a lens (d). The shuttered image is then projected to the camera (a) by a second lens (b). A controller (e) synchronizes LED panel and shutter wheel.

in the near-infrared range. It should be noted that the relatively long CCD readout time of $\sim 0.4 \mathrm{~s}$ does not limit the obtainable time-resolution, which is rather determined by the frequency of the shutter wheel than by the camera frame rate.

The shutter wheel is rotated by a brushless DC motor, which is driven by the excitation controller to obtain a shutter period $T$ between $250 \mu$ s and $10 \mathrm{~ms}$. The excitation controller synchronizes to the shutter wheel using a phaselocked-loop and switches the LED panel with an adjustable phase delay and duty cycle. This periodic modulation of the generation rate $G$ leads to a periodic $\Delta n$ in the sample. The corresponding PL emission $\Phi(t)$ is then modulated by the shutter wheel. The light reaching the camera sensor through the slits of the shutter wheel is therefore emitted in a specific time interval $t_{1} . . t_{2}$ of the periodic excitation cycle, which is determined by the phase delay $\varphi$ between the shutter wheel and excitation.

During the measurement, the LED panel is switched on at $t=0$ for half the period $T$. The actual shape of the LED emission curve differs a little from this theoretical rectangular function, as the driver electronics and LEDs have finite rise and fall times. The slopes of the LED intensity were measured with a $\mathrm{Si}$ photodiode in reverse bias. With the present setup, slopes shorter than $1 \mu \mathrm{s}$ (10\% to $90 \%$ ) are possible, so for effective lifetimes $>10 \mu \mathrm{s}$, this effect does not constitute a significant source of error.

The steady-state value $\Delta n_{0}$ under illumination with a constant generation rate $G$ is given by

$$
\Delta n_{0}=\lim _{t \rightarrow \infty} \Delta n=G \tau_{\mathrm{eff}} .
$$

As the following evaluation algorithm allows for nonequilibrium values of $\Delta n$ at the excitation flanks (see Eq. (5)),

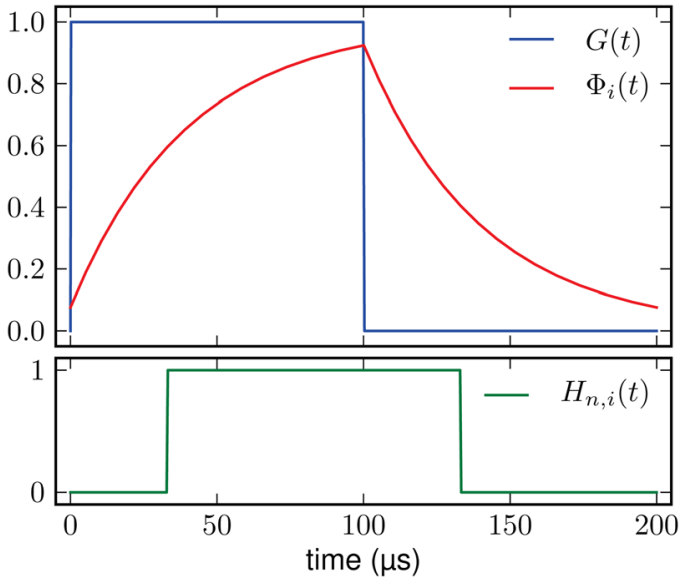

FIG. 2. (Color online) Model of the normalized generation rate $G(t)$ and PL intensity $\Phi_{i}(t)$ for a period of $T=200 \mu \mathrm{s}$ and $\tau_{\text {eff }}=40 \mu \mathrm{s}$. The bottom plot shows the shutter wheel transmit function $H_{n}(t)$ for an arbitrary phase delay $\varphi_{n}$.

the quasi-steady-state condition of $T \gg \tau_{\text {eff }}$ does not have to be satisfied. Best results were achieved with a period length $T$ of about 2-5 times the highest effective lifetime in the sample.

The camera then records several images $I_{n}$ with different phase delay values $\varphi_{n}$.

Taking advantage of the rectangular transmit function $H(t)$ of the shutter wheel (see Fig. 2), the camera signal $I_{n, i}$ at pixel $i$ is given by the integral

$$
I_{n, i}=\int_{0}^{T} \Phi_{i}(t) H(t) d t=\int_{t_{1}(n, i)}^{t_{2}(n, i)} \Phi_{i}(t) d t,
$$

where $t_{1}=\left(\varphi_{n}+\theta_{i}\right) T / 2 \pi$ and $t_{2}=t_{1}+T / 2$. The constant $\theta_{i}$ is an offset due to the physical size of the intermediate image and depends on the pixel position $i$.

\section{TIME-DEPENDENT PHOTOLUMINESCENCE}

To directly determine the time-dependent luminescence $\Phi_{i}(t)$ from the measured intensities $I_{n, i}$, the convolution integral in Eq. (2) has to be deconvoluted. Effective lifetime values can then be calculated from the slope of this transient PL curve. However, due to the limited number of measurement points and the noise of the PL images, this approach is difficult to realize. The numerical deconvolution algorithms we applied were prone to oscillatory artifacts and did not yet produce useful results.

An alternative approach is the comparison and fitting of $I_{n, i}$ values with a model of the expected intensities depending on $\tau_{\text {eff }}$ and other parameters. A suitable model was developed and shall be discussed below.

The thickness-averaged generation rate in the sample

$$
G(t)= \begin{cases}E(1-R) / w & \text { for } t<T / 2 \\ 0 & \text { for } t>T / 2\end{cases}
$$

depends on the incident photon flux $E$, the thickness of the sample $w$ and the reflectivity $R$ at the illumination wavelength. Transmission of excitation light was neglected in this model because of the short absorption length of about $3 \mu \mathrm{m}$ in silicon. Thickness-averaged values for $G$ and $\Delta n$ were 
used rather than depth-dependent quantities, which is a significant simplification. However, numerical simulations showed that the transient of $\Delta n$ is mainly unaffected by this depth-dependency for realistic values of surface recombination velocity and bulk lifetime. As the absolute value of $\Delta n$ is not relevant for the evaluation of $\tau_{\text {eff }}$, the simplification may be considered acceptable in this case.

The basic differential equation for the excess carrier density $\Delta n$ is

$$
\frac{d \Delta n}{d t}=G-\frac{\Delta n}{\tau_{\mathrm{eff}}} .
$$

In general, $\tau_{\text {eff }}$ depends on the density of excess charge carriers $\Delta n$, so the solution to Eq. (4) may become quite complicated. For multicrystalline wafers and the measurement setup presented in this work, $\Delta n$ is, in all cases, much lower than the net doping concentration $N$. Under these low level injection conditions, a constant $\tau_{\text {eff }}$ may be assumed, which leads to a monoexponential solution for $\Delta n(t)$ :

$$
\Delta n(t)=\left\{\begin{array}{cc}
\Delta n_{0}\left(1-f \exp \left(-\frac{t}{\tau_{\text {eff }}}\right)\right) & \text { for } t<T / 2, \\
\Delta n_{0}\left(f \exp \left(-\frac{t-T / 2}{\tau_{\text {eff }}}\right)\right) & \text { for } t>T / 2
\end{array}\right.
$$

where $\Delta n_{0}$ is the excess carrier density under steady-state illumination and $f$ is a factor corresponding to the "charge carrier saturation" $\left(\max (\Delta n(t)) / \Delta n_{0}\right)$ of the sample in the excitation phase. It can be determined by solving Eq. (5) for periodic boundary conditions:

$$
f=\frac{1}{1+\exp \left(-T / 2 \tau_{\text {eff }}\right)} .
$$

The assumption of periodic boundary conditions $\Delta n(0)$ $=\Delta n(T)$ is valid for the long-term steady-state after several excitation cycles, when the amount of generated charge carriers during an excitation cycle is equal to the number of recombinations in the same time.

The injection-dependant PL intensity $\Phi$ at room temperature is given by

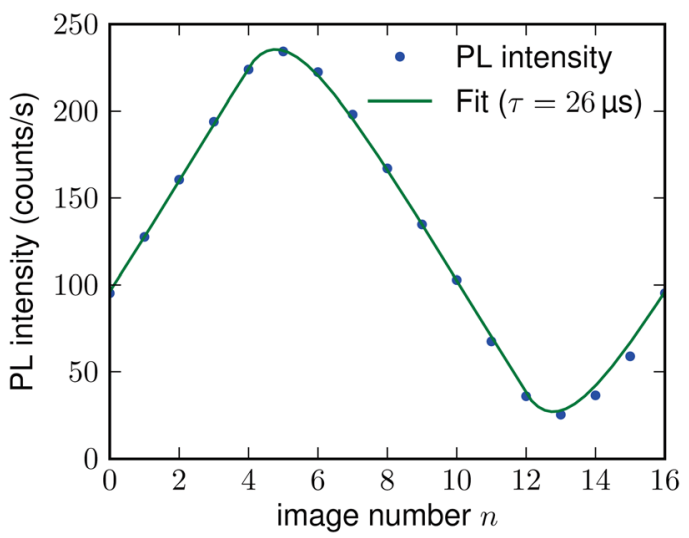

FIG. 3. (Color online) PL intensity curve $I_{n, A}$ for a spot with high lifetime (marked as A in Fig. 5) and the corresponding fit $I_{n, A}^{*}$. The carrier lifetime $\tau_{\text {eff }}$ determines the curvature of $I^{*}$ at the turning points.

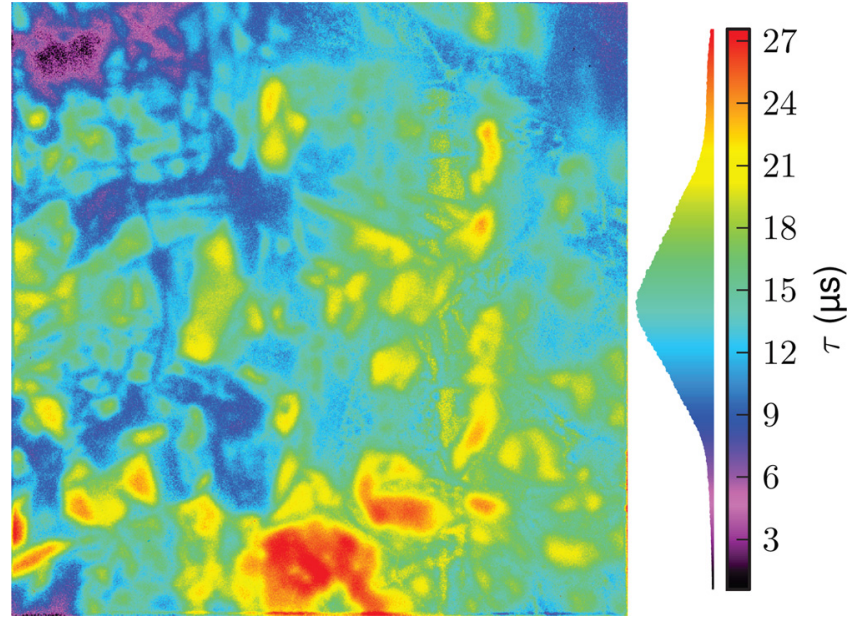

FIG. 4. (Color online) Lifetime image of a $\mathrm{SiN}_{x}$-passivated $5 \mathrm{~cm}$ wide $\mathrm{mc}-\mathrm{Si}$ wafer measured with the TR-PLI method at $E=2.5 \times 10^{17}$ phot. $/ \mathrm{cm}^{2} \mathrm{~s}$ and $T \approx 350 \mu \mathrm{s}$.

$$
\Phi(\Delta n)=B \Delta n(N+\Delta n),
$$

where $N$ is the doping concentration and $B$ is the coefficient of radiative recombination. $B$ is constant except for high values of $\Delta n,{ }^{15}$ which do not occur in the multicrystalline wafers examined in this work. Using the time-dependent excess carrier density from Eq. (5), we obtain a PL intensity $\Phi(t)$, which can be convoluted analytically according to Eq. (2). The resulting intensity model $I_{n, i}^{*}$ (see Appendix A) can be fitted to the data $I_{n, i}$ with the parameters $\tau_{i}$ and a camera sensitivity factor $C_{i}$ for each pixel (see Fig. 3).

\section{EXPERIMENTAL RESULTS}

Lifetime measurements performed with this method on passivated multicrystalline silicon wafers are in good agreement with steady-state PL images. Figure 4 shows a TR-PLI measurement of a $\mathrm{SiN}_{x}$-passivated $5 \mathrm{~cm}$ wide p-type mc-Si wafer with a thickness of about $215 \mu \mathrm{m}$. The irradiation from the LED panel was $E=2.5 \times 10^{17}$ phot./ $\mathrm{cm}^{2}$ s, leading to a maximum injection level of about $3 \times 10^{14} \mathrm{~cm}^{-3}$ in the high-

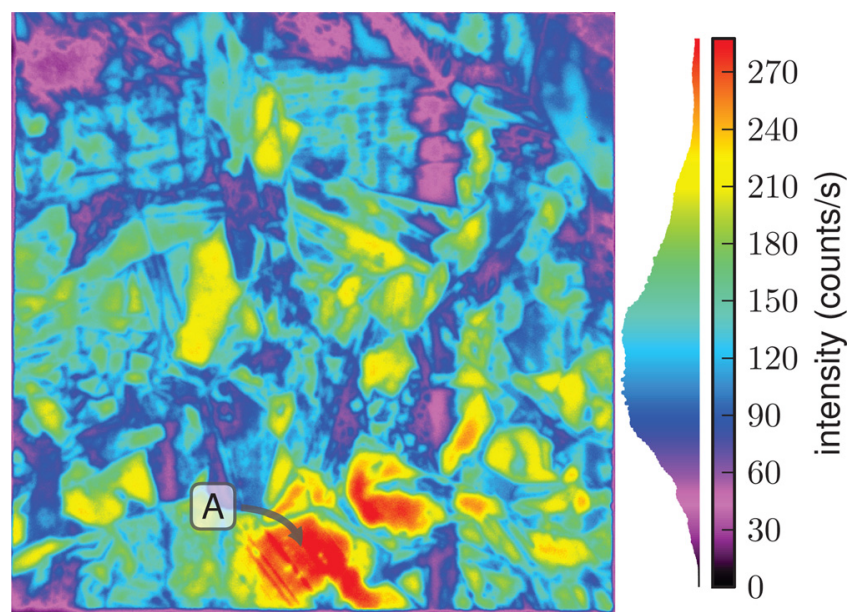

FIG. 5. (Color online) Steady-state PL image of the wafer shown in Fig. 4. 

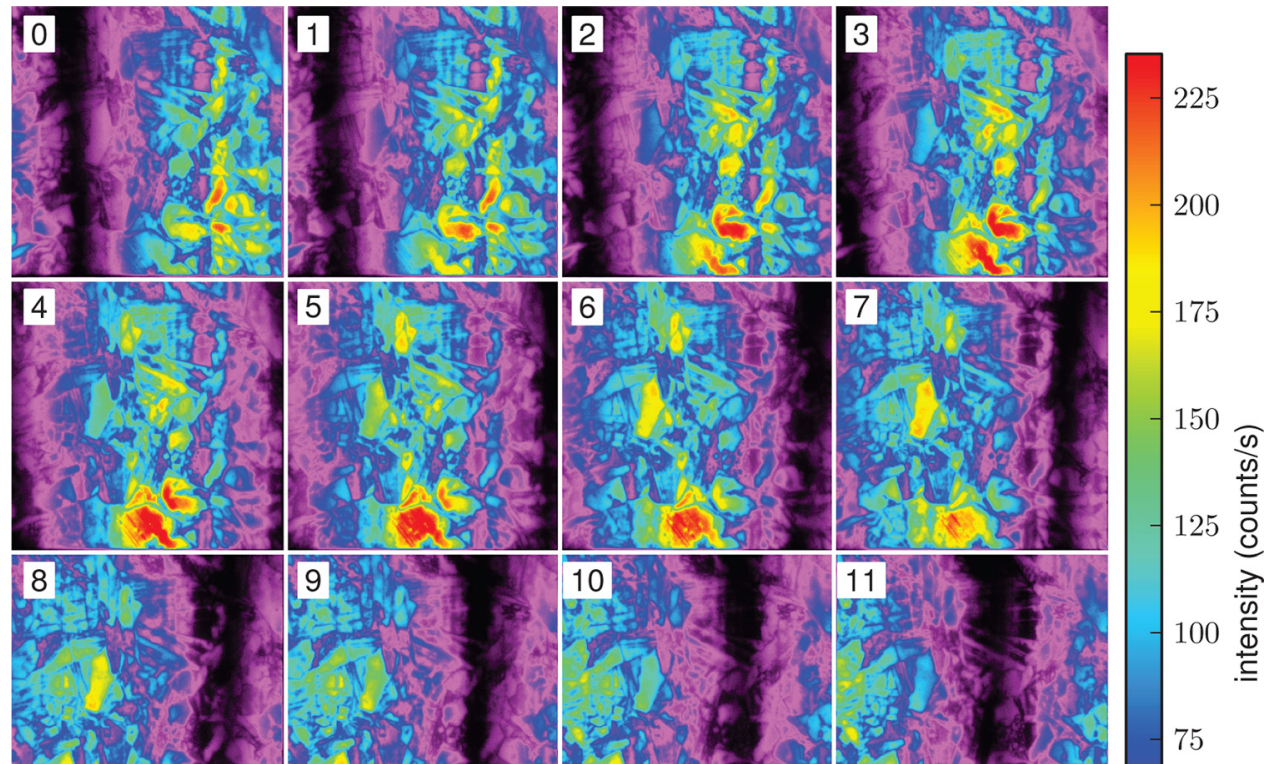

FIG. 6. (Color online) The 16 PL images recorded for the TR-PLI lifetime map shown in Fig. 4. Each picture is taken at an increasing phase shift $\varphi_{n}=2 \pi \frac{n}{16}$ between shutter wheel and excitation. The occlusion effect of the shutter wheel can be seen very well in these images: the darker region (where PL light passes to the camera only during the non-excited half of the period) moves from right to left, corresponding to the direction of shutter wheel rotation.

lifetime regions. The $16 \mathrm{PL}$ images recorded for this measurement are shown in Fig. 6. Each image has an exposure time of $20 \mathrm{~s}$, and the total measurement time was $\sim 5$ minutes.

The variation of $\varphi$ over the image series can be seen in the horizontal shift of the intensity gradient in the image as well as the influence of $\theta_{i}$, which is responsible for this gradient (see Eq. (2)).

A steady-state PL image of the wafer is shown in Fig. 5. It shows very good qualitative agreement to the lifetime image in Fig. 4. The label A marks the position in a grain with high lifetime, where the intensity values for Fig. 3 were taken. The effect of a high effective lifetime on the PL images can be seen directly in Fig. 6; in image number 13, the values of $\varphi_{13}$ and $\theta_{A}$ (see Eq. (2)) for position A lead to an integration of only the non-illuminated part of the transient PL emission curve $\Phi(t)$. The same is true for all other pixels on a vertical line with the same $\theta_{i}$. As can be seen, the remaining PL intensity $I_{13, A}$ of position $\mathrm{A}$ in image 13 is much higher than the intensity in the areas above it. This is caused by the slower decay of excited charge carriers in A, leading to a higher value of the integral $I_{13, A}$.

A notable difference between steady-state PL images and the TR-PLI lifetime maps can be observed in respect to contrast. Due to internal reflections of PL radiation in the sample, a certain amount of blurring is present in PL images. This affects the transient measurement mainly in low-lifetime regions, where PL light from adjacent highlifetime areas may be emitted with the respective slow transient. ${ }^{3,12}$ The resulting overestimation of the effective lifetime can be seen, e.g., at grain boundaries. The meas- ured transient lifetime in good and average regions of the sample is mainly unaffected by this effect. A lifetime measurement with high contrast can therefore be obtained by calibrating the steady-state PL image with the TR-PLI lifetime in those regions, as proposed by Ramspeck et al. ${ }^{11,12}$ However, this requires a laterally homogeneous relation between lifetime and PL signal, which is not necessary for normal TR-PLI lifetime maps.

\section{QSSPC SENSITIVITY AND COMPARISON}

Reference measurements of the studied samples were done using the quasi-steady-state photo conductance (QSSPC) technique. The conductance of the sample is measured via eddy currents induced by a coil of an oscillator circuit. A change in conductivity, e.g., due to the decay of photogenerated charge carriers, alters the inductance of the coil directly beneath the sample and therefore shifts the resonance frequency and influences the amplitude of the oscillation. The coil used in the specific instrument is typically a short coil with a diameter of around $2 \mathrm{~cm}$.

To compare the results of the spatially resolved TR-PLI technique with the area-averaged QSSPC method, the contribution of local lifetime values to a global QSSPC measurement has to be known. The QSSPC measurements were carried out using the commercially available WCT-120 lifetime tester from Sinton Instruments operating in quasisteady-state mode. According to Cuevas, ${ }^{16}$ this device measures an area-weighted average of the present lifetimes appearing in the material located above the sensor region. 


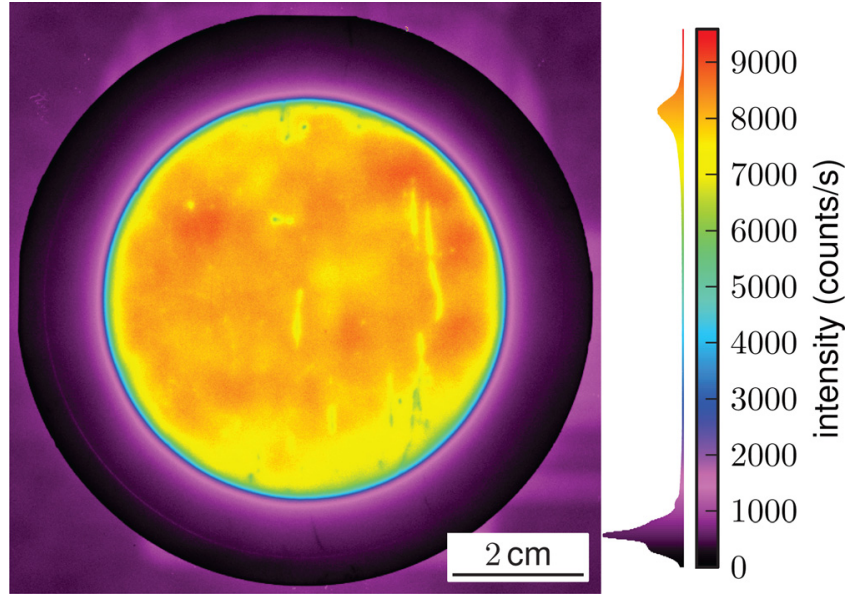

FIG. 7. (Color online) Steady-state PL image of a circular Cz-Si wafer with a partially ablated $\mathrm{SiN}_{x}: \mathrm{H}$ surface passivation layer. The inner region is not yet ablated and shows a high $\tau_{\text {eff }}$.

Unfortunately, the spatial sensitivity function of the sensor, which is responsible for the area-weighting, is unknown. However, in the case of multicrystalline materials used in this work, spatially very inhomogeneous lifetimes may occur, so that the knowledge about the area-weighting is essential for the correct lifetime calibration.

The sensitivity function of the QSSPC setup was measured assuming radial symmetry of the system. Circular monocrystalline Czochralski-grown $(\mathrm{Cz})$ silicon wafers passivated by amorphous hydrogenated $\mathrm{SiN}_{x}: \mathrm{H}$ on both sides were used. The samples exhibit laterally very homogeneous effective lifetimes of about $60 \mu \mathrm{s}$. To determine the radial contribution to the average lifetime value, concentric rings of the $\mathrm{SiN}_{x}: \mathrm{H}$ passivation layer have been removed by laser ablation to dramatically reduce the effective carrier lifetime in those areas down to about $2 \mu$ s (see Fig. 7). After each ablation step, the average lifetime $\tau_{\mathrm{PC}}$ was measured via QSSPC using the quasi-steadystate mode. As expected, the measured average lifetime decreases with lowered area of the remaining passivation layer. The results of this ablating procedure are shown in Fig. 8. Assuming $\tau_{\text {eff }}(r)$ is a step function changing from $\tau_{1}$ to $\tau_{2}$ at $r=r^{\prime}$ and $S(r)$ is a sensitivity function which depends

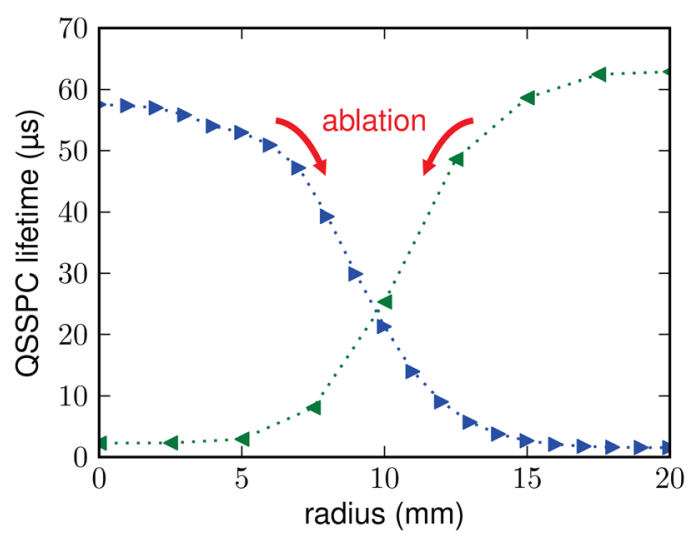

FIG. 8. (Color online) Varying lifetime of two circular Cz-Si wafers when removing the $\mathrm{SiN}_{x}$ :H passivation by laser irradiation annularly from the outside to the middle (markers pointing left) and from the middle to the outside (markers pointing right), respectively. only on the radius $r$, the integral quantity $\tau_{\mathrm{PC}}\left(r^{\prime}\right)$ can be expressed as an integral over the local effective lifetime $\tau_{\text {eff }}$ :

$$
\begin{aligned}
\tau_{\mathrm{PC}}\left(r^{\prime}\right) & =\int_{0}^{\infty} 2 \pi r S(r) \tau_{\mathrm{eff}}(r) d r \\
& =\int_{0}^{r^{\prime}} 2 \pi r S(r) \tau_{1} d r+\int_{r^{\prime}}^{\infty} 2 \pi r S(r) \tau_{2} d r .
\end{aligned}
$$

$S(r)$ may then be determined from the derivative of the measured lifetime $\tau_{\mathrm{PC}}(r)$ by the distance $r$ to the sensor center:

$$
S(r)=\frac{1}{2 \pi r\left(\tau_{1}-\tau_{2}\right)} \frac{d \tau_{\mathrm{PC}}}{d r} .
$$

The results of this calculation are depicted in Fig. 9. As can be seen, the inwards and the outwards ablating procedure each fit to Gaussian functions. The sensitivity curves of the ablating procedures exhibit radial shifts on the r-axis. These deviations may arise from laser-induced crystallographic defects and lateral carrier diffusion and depend on the direction of ablation. For determining the real sensitivity function, the mean curve of the two procedures was calculated in order to ensure that the contrary acting systematic errors cancel out each other. The resulting mean curve may then be approximated by a Gaussian function

$$
S(r)=A \exp \left(-\left(\frac{r-r_{0}}{\sigma}\right)^{2}\right),
$$

with $r_{0}=9.3 \mathrm{~mm}$ and $\sigma=3.6 \mathrm{~mm}$. The normalization factor $A$ derives from the integration in Eq. (7) with $\tau_{\mathrm{eff}}=\tau_{\mathrm{PC}}$.

As can be seen, the maximal sensitivity of the sensor is at a distance of $9.3 \mathrm{~mm}$ to the device center and is therefore located near the coil used for measuring the photo conductance. This enables correct lifetime comparison of TR-PL images and QSSPC measurements by calculating an average value of the PL lifetimes, weighted with the sensitivity function.

Giesecke et $\mathrm{al}^{3}$ have very recently published a measurement of the WCT-120 sensitivity function with a similar approach. The results agree quite well with our measurements.

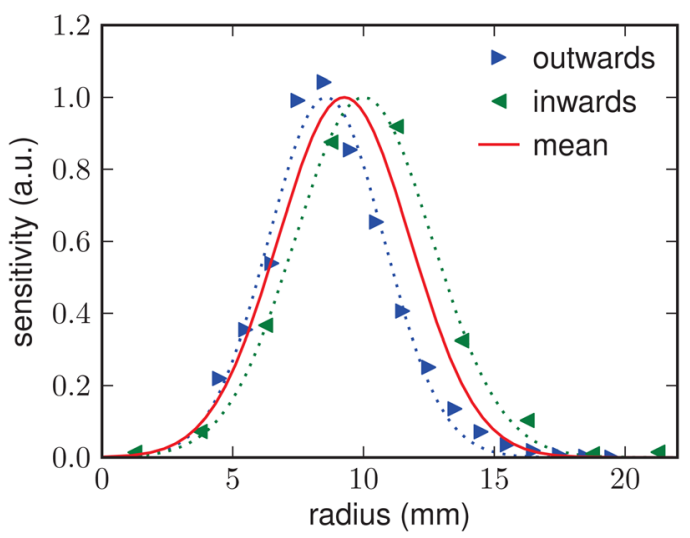

FIG. 9. (Color online) Radial sensitivity curves determined by the ablating procedures mentioned above. Due to crystallographic defects caused by the laser and excess carrier diffusion, the measured sensitivity curves are shifted toward the ablated region. When using the mean curve, these contrary acting systematic errors cancel out each other. 


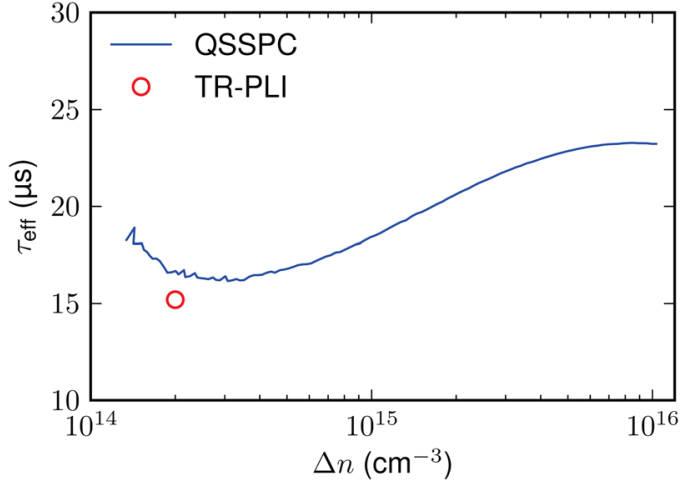

FIG. 10. (Color online) QSSPC lifetime measurement of the sample shown in Fig. 4. The weighted average lifetime value from TR-PLI is shown by the circle.

It has to be noted that Eq. (7) is only valid for photoconductance measurements in quasi-steady-state mode. In transient mode, the different photoconductance decay curves of the sensitive area combine to an integral transient curve, which usually overestimates the average effective lifetime significantly, depending on the evaluation method. Photoconductance measurements in transient mode showed that effective lifetime values of very inhomogeneous samples mostly correspond to the maximum effective lifetime in the sensitive area.

An average lifetime value of the TR-PLI measurement shown in Fig. 4 was calculated using this calibration function. The resulting value $\tau_{\text {eff, avg }}=15.2 \mu$ s agrees very well with QSSPC measurements of the sample, which can be seen in Fig. 10. As the LED panel of the PL setup can reach a light intensity of about one sun equivalent, the maximum excess carrier density is lower for TR-PLI than for QSSPC, which uses a single high-power flash for excitation. The average value of $\Delta n$ for the measurement shown in Fig. 4 can be estimated with Eqs. (1) and (3) to about $2 \times 10^{14} \mathrm{~cm}^{-3}$. At this level, trapping effects are beginning to show in the QSSPC curve, leading to an overestimated effective lifetime value. ${ }^{8}$ Photoluminescence imaging is not affected by this trapping, ${ }^{1}$ which explains the slightly lower value of the TR-PLI method (circle in Fig. 10).

\section{CONCLUSIONS}

In this work, a fast, camera-based method for spatially and time-resolved measurement of photoluminescence in crystalline silicon wafers has been presented. Typical measurement time for a lifetime image of a multicrystalline wafer at 1 megapixel resolution is $1-5$ minutes, whereas a MW-PCD measurement with the same resolution takes several hours. The transient measurement makes the PL image independent of external calibration and reduces the influence of lateral inhomogeneities. Effective lifetimes down to less than $10 \mu$ s can be measured with the current setup and evaluation algorithm. Compared with the dynamic ILM/CDI method, ${ }^{11}$ the spatial resolution of the TR-PLI measurement is much higher. The focus of this work lies on multicrystalline silicon wafers with comparably low $\tau_{\text {eff }}$ to demonstrate the potential of the TR-PLI method. Good agreement between QSSPC measurements and properly averaged TR-PLI lifetimes could be obtained.

\section{ACKNOWLEDGMENTS}

The financial support of the German BMU project 325033 is gratefully acknowledged. The content of this publication is the responsibility of the authors.

\section{APPENDIX A: ANALYTICAL SOLUTION OF THE INTENSITY MODEL}

Due to the periodicity of the model, the solution was calculated in angular space with $x=\frac{2 \pi}{T} t$ and $\tau=\frac{2 \pi}{T} \tau_{\text {eff }}$ :

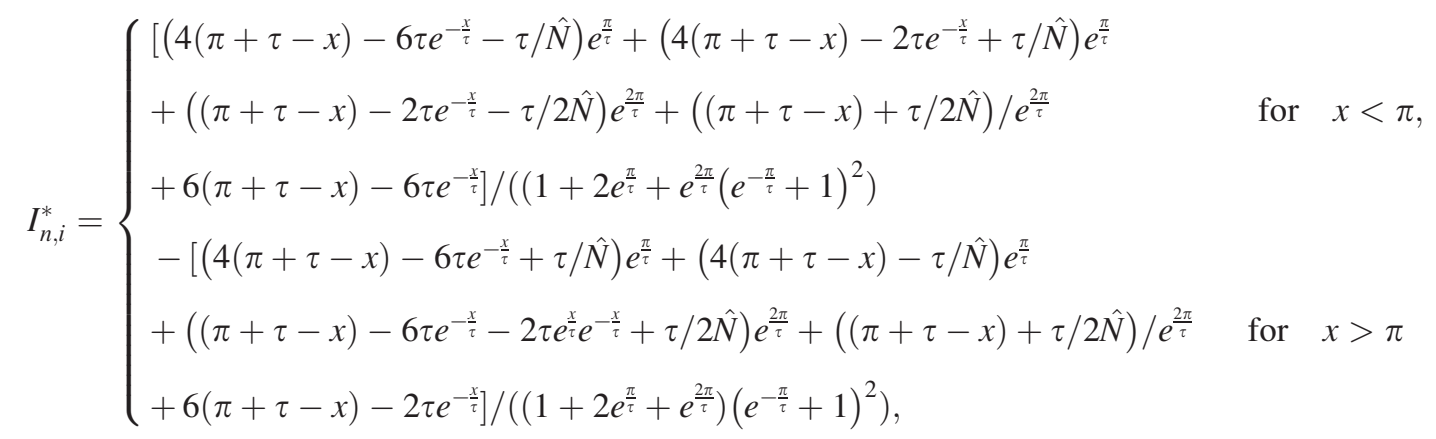

where $\hat{N}=\frac{N}{\Delta n_{0}}+1$ derives from the doping concentration $N$.

${ }^{1}$ T. Trupke, R. A. Bardos, M. C. Schubert, and W. Warta, Appl. Phys. Lett. 89, 044107 (2006).

${ }^{2}$ S. Herlufsen, J. Schmidt, D. Hinken, K. Bothe, and R. Brendel, Phys. Status Solidi (RRL) 2, 245 (2008).

${ }^{3}$ J. A. Giesecke, M. C. Schubert, B. Michl, F. Schindler, and W. Warta, Sol. Energy Mater. Sol. Cells 95, 1011 (2010).

${ }^{4}$ D. Hinken, K. Bothe, K. Ramspeck, S. Herlufsen, and R. Brendel, J. Appl. Phys. 105, 104516 (2009).

${ }^{5}$ D. Macdonald, J. Tan, and T. Trupke, J. Appl. Phys. 103, 073710 (2008).
${ }^{6}$ P. P. Altermatt, F. Geelhaar, T. Trupke, X. Dai, A. Neisser, and E. Daub, Appl. Phys. Lett. 88, 261901 (2006).

${ }^{7}$ J. A. Giesecke, M. C. Schubert, D. Walter, and W. Warta, Appl. Phys. Lett. 97, 092109 (2010)

${ }^{8}$ R. A. Sinton, A. Cuevas, and M. Stuckings, Conference Record of the 25th IEEE Photovoltaic Specialists Conference, Washington, DC, 13-17 May 1996 (IEEE, New York, 1996), pp. 457-460.

${ }^{9}$ T. Trupke, R. A. Bardos, and M. D. Abbott, Appl. Phys. Lett. 87, 184102 (2005).

${ }^{10}$ J. Schmidt and A. G. Aberle, J. Appl. Phys. 81, 6186 (1997).

${ }^{11}$ K. Ramspeck, S. Reissenweber, J. Schmidt, K. Bothe, and R. Brendel, Appl. Phys. Lett. 93, 102104 (2008). 
${ }^{12}$ K. Ramspeck, K. Bothe, J. Schmidt, and R. Brendel, J. Appl. Phys. 106, 114506 (2009).

${ }^{13}$ S. Herlufsen, K. Ramspeck, D. Hinken, A. Schmidt, J. Müller, K. Bothe, J. Schmidt, and R. Brendel, Phys. Status Solidi (RRL) 5, 25 (2011).

${ }^{14}$ D. Kiliani, G. Micard, B. Raabe, and G. Hahn, Proceedings of the 25th EU PVSEC, Valencia, Spain, 6-10 September 2010, pp. 1363-1366.
${ }^{15}$ P. P. Altermatt, F. Geelhaar, T. Trupke, X. Dai, A. Neisser, E. Daub, Proceedings of the 5th International Conference on Numerical Simulation of Optoelectronic Devices (IEEE), Berlin, Germany, 19-22 September 2005 2, 47 (2005).

${ }^{16} \mathrm{~A}$. Cuevas, paper presented at the 8th Workshop on Crystalline Silicon Solar Cells Materials and Processes, Copper Mountain Resort, CO, 17-19 August 1998. 